\title{
New ideas on the pathophysiology and treatment of lung disease
}

\author{
D F Rogers, G J Laurent
}

\begin{abstract}
There is a continuing need for development of new treatments for lung disease. Basic scientific investigations are identifying novel targets for the development of new approaches to therapy of a range of respiratory conditions. Coupled with the advances in technology being harnessed by the pharmaceutical and biotechnological industries, there is now an impressive range of potential treatments including gene therapy, not just for cystic fibrosis but also for a range of inflammatory lung conditions, anti-cytokine and antiadhesion molecule approaches, and targeting of intracellular signal transduction pathways including cyclic AMP metabolism, tyrosine kinases and MAP kinases. "Old" molecules such as heparin and secretory leukoprotease inhibitor (SLPI) are demonstrating new beneficial activities. Simple molecules such as nitric oxide (NO) gas may be involved in the pathophysiology of different airway conditions. It is an exciting time for respiratory science and a time for optimism for those seeking new approaches to the treatment of lung diseases.

(Thorax 1998;53:200-203)
\end{abstract}

Keywords: Science Matters; review; asthma; lung disease; pulmonary fibrosis; gene therapy; airway inflammation

Science Matters finishes this month after a two year run. During that time we have been treated to a range of short articles with new viewpoints on lung diseases from asthma and ARDS to pulmonary fibrosis and hypertension. Each article showed that science mattered in understanding pathophysiology and in determining rational approaches to therapy. Because of the diversity of the articles we thought it would be helpful to new readers of Thorax, and as an aide mémoire to regular readers, to collate and review the articles to identify unifying themes. We also take this opportunity to thank all our contributors for their sterling efforts in adhering to our editorial demands for the short focused articles which made the series accessible to a wide audience. We also thank our specialist reviewers for ensuring scientific accuracy and our group of informedbut-non-specialist referees who ensured the "readability" of the articles.

\section{Gene therapy}

Gene therapy is the newest approach to treatment of lung disease. The rationale behind somatic gene therapy is the replacement, modification, or suppression of the gene(s) considered responsible for the pathophysiology of a given clinical condition. The lung provides a particularly attractive target for gene therapy because of its accessibility through the airways or the vasculature. Alpha $a_{1}$-antitrypsin deficiency and cystic fibrosis are the two most common fatal single gene defects involving the lung in Western communities. The genetic defect in both conditions is well characterised which has led to these diseases being the front runners in lung directed gene therapy. In cystic fibrosis four "preliminary" studies have so far been published on topical delivery of the CFTR (cystic fibrosis transmembrane conductance regulator) gene to the airways, with more reported successes than failures in correcting the transepithelial chloride $\left(\mathrm{Cl}^{-}\right)$ transport defect. ${ }^{1}$ Progress is steady and encouraging. Problems with existing vectorsthe inflammatory but efficient adenoviral vectors versus the less efficient but safer cationic liposome vectors-may be overcome by new vectors such as adeno-associated virus or viral liposomes. Experimental studies indicate that only about $8 \%$ of cells need to be "corrected" to counter the $\mathrm{Cl}^{-}$transport defect, and only $5 \%$ of normal CFTR mRNA needs to be expressed to increase $\mathrm{Cl}^{-}$transport markedly. Re-evaluation of a number of assumptions, including alternative end points to $\mathrm{Cl}^{-}$ transport (such as bacterial adherence) and the optimal target for gene delivery (surface epithelium versus submucosal glands), should focus future studies with a view to hastening the advent of gene therapy for cystic fibrosis. The advances made in gene therapy for cystic fibrosis and other single gene defect conditions have prompted interest in the possibility of similar therapy for more complex pulmonary conditions characterised by lung inflammation. ${ }^{2}$ Although seemingly less amenable than cystic fibrosis to gene therapy, chronic obstructive pulmonary disease (COPD), adult respiratory distress syndrome (ARDS), and asthma could be targets for beneficial genetic modification of critical elements of the inflammatory process. Inflammatory lung conditions result from an imbalance between injurious inflammatory mediatorsfor example, neutrophil elastase and reactive oxygen species - and endogenous cytoprotective mechanisms. Genetic intervention to redress the balance might therefore prove effective therapy. For example, overexpression of anti-elastase genes could limit elastase mediated lung damage in emphysema. Overexpression of genes encoding anti-inflammatory cytokines such as IL-10 and of inhibitors of 
"inflammatory" transcription factors-for example, I $\mathrm{I} \mathrm{B} \alpha$, the inhibitor of $\mathrm{NF}-\kappa \mathrm{B}$-are other examples. The effectiveness of these treatments in inflammatory lung disease will be dependent, as is gene therapy for cystic fibrosis, on selection of the appropriate cellular target, an enormous challenge in itself, as well as on refinements in delivery vectors, suppression of host immune response, cell specificity and nuclear targeting, and on clinical end point selection.

\section{Airway inflammation}

Asthma is now viewed as an inflammatory airway disease and may even be specifically redefined as an IL- 5 driven eosinophilic bronchitis. ${ }^{3}$ Inhalation of allergen and the subsequent allergic reaction in the lung leads to the production of antibody and cytokines, including IL-5. IL-5 is carried in the blood to the bone marrow where it stimulates eosinophil production. Eosinophils migrate to the lung where they cause tissue damage resulting in asthma. Consequently, inhibition of IL-5 production-for example, by specific targeting of the IL-5 promoter or blocking of the effects of IL-5 by development of receptor antagonists - could be fundamental therapeutic options in asthma. The simple gas nitric oxide (NO) is increased in the exhaled air of asthmatic patients compared with controls, presumed to be a reflection of airway inflammation, and is implicated in the pathophysiology of asthma. ${ }^{4} \mathrm{NO}$ is generated from L-arginine by three forms of $\mathrm{NO}$ synthase, two of which are constitutive (cNOS) and one inducible (iNOS). The suggestion is that the NO generated by cNOS is beneficial in local airway physiological control, but that the much larger amounts of NO generated by iNOS are detrimental by amplifying the inflammatory response. Consequently, selective iNOS inhibitors could have therapeutic anti-inflammatory potential in asthma and such drugs are currently in development.

The involvement of airway sensory nerves in asthma has been a burning issue for over a decade, and the debate continues. ${ }^{5}$ C-fibres (sensory nerves) which are sensitive to capsaicin, the pungent principle of hot peppers, contain the peptide neurotransmitters substance $P$ (SP), neurokinin A (NKA), and calcitonin gene-related peptide (CGRP). Laboratory experiments have shown that activation of these nerves or administration of sensory neuropeptides reproduces many aspects of the asthmatic reaction including bronchoconstriction, vasodilatation, plasma exudation and mucus secretion, as well as effects on inflammatory cells. There is some evidence that sensory nerves, sensory neuropeptides, and receptors for SP and NKA (tachykinin receptors) are increased in asthmatic airways. Drugs which inhibit sensory nerve activity could therefore be beneficial in asthma. Tachykinin receptor antagonists are already in clinical trial for asthma, with mixed results. Drugs with a broader spectrum of inhibitory action on airway nerves, including cholinergic nerves, may have greater therapeutic potential not only in asthma but in other "neurogenic" airway conditions such as chronic bronchitis and rhinitis. For example, drugs which open potassium $\left(\mathrm{K}^{+}\right)$channels inhibit nerve activity. ${ }^{6}$ In particular, the large conductance calcium activated $\mathrm{K}^{+}$channel $\left(\mathrm{BK}_{\mathrm{Ca}}\right)$ mediates the inhibition of a number of neurally active drugs. Selective openers of this channel may therefore inhibit neurogenic responses in asthma and chronic bronchitis, and also have the added benefit of being bronchodilators. Phosphodiesterase (PDE) inhibitor drugs are also bronchodilators because they block the action of endogenous PDE and limit the breakdown of intracellular cAMP. ${ }^{7}$ The accumulated cAMP induces relaxation of airway smooth muscle. Importantly, PDE inhibitors also have multiple anti-inflammatory actions which, together with their bronchodilator properties, would make them a panacea for asthma. The challenge to drug development at present is that PDEs comprise seven families of isoenzymes, some of which include multiple proteins encoded by distinct genes. PDE- 3 and PDE-4 are most relevant to the airways and clinical trials using dual PDE-3/4 inhibitors to maximise bronchodilator and antiinflammatory activity are underway. Unfortunately, results to date are equivocal and unwanted side effects, most notably nausea and emesis, are prevalent. The hope is that there may be an airway specific PDE isoform against which PDE inhibitor drugs without side effects could be developed.

Glucocorticosteroids are mainstay antiinflammatory therapy in asthma. In common with many therapeutic agents, their precise mechanism of action is incompletely defined but involves suppression of proinflammatory mediators and upregulation of antiinflammatory proteins, one of which is lipocortin $1 .^{8}$ Lipocortin 1 mediates the antiinflammatory effects of glucocorticosteroids and in experimental systems has antiinflammatory effects. Consequently, inhaled lipocortin 1 could have therapeutic potential, although there are problems with instability and bioavailability. Presently available synthetic peptides are less potent than lipocortin 1 and will need to be superseded by compounds which retain the biological activity of the parent molecule. A more specific approach to halting influx of inflammatory cells into the airway interstitium is to prevent leucocytes from leaving the vascular compartment. ${ }^{9}$ Selectins are adhesion receptors on leucocytes and endothelial cells which mediate the initial binding of the leucocytes to the venular endothelium. Binding is the essential first step in leucocyte migration into the airway tissue. Prevention of leucocyte emigration could be useful in the treatment of a number of airway conditions including fibrosing alveolitis and asthma. Development of effective selectin antagonists without immunosuppressive activity is the hurdle to overcome. The mast cell product and anticoagulant heparin is recognised as having anti-inflammatory potential, including inhibition of leucocyte trafficking across the endothelium. ${ }^{10}$ The latter activity is due to an action on $\mathrm{T}$ cell heparinase rather 
than an antiselectin action. Inhaled heparin inhibits experimentally induced asthmatic responses whilst topical administration inhibits nasal inflammation. Heparin-like molecules lacking anticoagulant activity could be antiinflammatory and also limit airways remodelling. From the above, there are many possibilities for new treatments for asthma and other inflammatory lung diseases. It should be noted, however, that investigation of airway epithelial function and of the repair processes involved after damage and shedding has led to the suggestion that any therapy for asthma needs to include epithelial cytoprotective activity. ${ }^{11}$

In respiratory distress syndrome (RDS) surfactant replacement is highly effective in treatment of premature infants. ${ }^{12}$ In contrast, surfactant replacement therapy in ARDS has not been so successful, despite predictions that it would be. The problem is that the clinical studies performed thus far have been limited in their design and lack the power to determine whether or not surfactant therapy is useful in ARDS. A prominent confounding aspect is the unique composition of the surfactant of individual patients. Identification of patients at risk, from serum levels of surfactant proteins $A$ and $\mathrm{B}$, with subsequent prophylactic delivery of nebulised, complementary, "tailor made" surfactant is required in future clinical investigations of the usefulness of surfactant replacement therapy in ARDS.

Bronchoalveolar lavage (BAL) has become a routine clinical procedure for monitoring of lung inflammatory indices in a variety of conditions including allergic disease, asthma, and during intensive care. ${ }^{13}$ However, the composition of BAL fluid is only a photographic snapshot in time and is affected by a number of factors. For example, the number of lymphocytes in BAL fluid is dependent upon the rate of entry into, persistence in (local proliferation versus apoptosis), and exit from the bronchoalveolar space. Understanding of lymphocyte kinetics will further our understanding of the pathophysiology of many inflammatory lung diseases.

Secretory leukoprotease inhibitor (SLPI) SLPI is another molecule-this time an endogenous one synthesised by the pulmonary epithelium-which, like PDE inhibitors, has multiple activity. Firstly, in partnership with $\alpha_{1}$-proteinase inhibitor $\left(\alpha_{1}-\mathrm{PI}\right)$, SLPI is recognised as a major component of the antineutrophil elastase shield in the lung. ${ }^{14}$ SLPI also has anti-HIV activity and is a broad spectrum antibiotic with antiretroviral, bactericidal, and antifungal activity. ${ }^{15}$ Consequently, inhaled recombinant SLPI (rSLPI) could have widespread therapeutic potential in lung diseases in which neutrophils are implicated in the aetiology (including emphysema, chronic bronchitis, cystic fibrosis, and ARDS), and as alternative treatment for infection, especially where drug resistance is limiting the usefulness of established antibiotics.

\section{Pulmonary hypertension}

Pulmonary hypertension may be a primary disease or a sequel to other cardiopulmonary conditions, in particular chronic hypoxic lung disease. In contrast to asthma, where increased NO production is implicated in pathophysiology (see above), reduced endothelial NO production may contribute to pulmonary vasoconstriction and vascular remodelling in pulmonary hypertension. ${ }^{16}$ Clinical studies have shown that inhaled NO is an effective and airway selective vasodilator in various forms of pulmonary hypertension, although investigations into the toxicity of $\mathrm{NO}$ are required prior to widespread and prolonged use. The vascular remodelling may be the result of growth factors which are endothelium-derived vasoconstrictors, in particular endothelin-1 and plateletderived growth factor. ${ }^{17}$ Whatever the growth factor, the suggestion is that there are common intracellular signal transduction mechanisms which convert hypoxia into cell replication. Identification of these pathways - whether involving phosphoinositide metabolism, protein kinase C (PKC), tyrosine kinases, or mitogenactivated protein kinases (MAP kinases) - and their inhibition by selective drugs might interrupt the vicious circle between hypoxic vasoconstriction and vascular remodelling.

\section{Pulmonary fibrosis}

Diffuse (interstitial) lung diseases including pulmonary fibrosis are triggered by diverse factors including therapeutic drugs and exposure to inorganic dusts or radiation. However, not all exposed individuals develop fibrosis, which indicates a genetic predisposition. ${ }^{18}$ Genetic polymorphisms and their risk factors for development of pulmonary fibrosis are emerging. Identification of patients with genetic susceptibility would, theoretically, allow improved monitoring of disease progression and the initiation of early, and therefore most effective, therapeutic intervention. One novel approach to the treatment of pulmonary fibrosis is anticytokine therapy. ${ }^{19}$ The challenge to anticytokine drug development is that a number of cytokines are likely to be involved in the development of fibrosis, including TGF- $\beta_{1}$ and TNF- $\alpha$, as well as blood-borne mediators and products of the coagulation cascade. Possible solutions are to block the action of multiple cytokines simultaneously, or inhibit the actions of several cytokines at different stages of the disease process or, as in the approach to vascular remodelling above, target an intracellular pathway common to a number of cytokines of interest. A variety of anticytokine drugs are available-for example, decorin, a natural TGF- $\beta$ inhibitor - and clinical trials with rational end points are merited. Another approach is to inhibit interactions between lung cells and extracellular matrix..$^{20}$ Lung cells and their surrounding matrix play more than mere structural and mechanical roles and, instead, interact dynamically via cell surface receptors, in particular the integrins, and specific matrix elements. The interactions lead to intracellular signal transduction, including ras, PKC and MAP kinase pathways, leading to 
cytoskeletal reorganisation, cell spreading, and migration. Drugs aimed at interfering with these interactions-for example, integrin blocking peptides-might have therapeutic potential in pulmonary fibrosis.

\section{Concluding remarks}

Articles from international contributors to the Science Matters series have highlighted how respiratory science in its many facets is flourishing and identifying a range of possible targets for new approaches to treatment of lung disease. These advances, coupled with the technology being harnessed by the pharmaceutical and biotechnological industries, will provide a range of pharmacological and immunological tools. Developments in the "reductive" biological sciences (molecular biology and cellular chemistry) and the continuance of investigations in complex in vivo systems ${ }^{21}$ is a powerful combination with which to test novel therapeutic compounds. Only a very few of these will emerge as new drugs in respiratory clinics. Those that do make it to the market place will add to or replace current drugs which, although effective in many instances, are so often inadequate. It is an exciting time for respiratory science and one for optimism for those of us who wish to see new approaches to the treatment of lung disease.

1 Middleton PG, Alton EWFW. Gene therapy for cystic fibrosis: which postman, which box? Thorax 1998;53:197-9.
2 Sallenave J-M, Porteous DJ, Haslett C. Gene therapy for Sallenave J-M, Porteous DJ, Haslett C. Gene therapy for lung inflam

3 Singh AD, Sanderson CJ. Anti-interleukin 5 strategies as a potential treatment for asthma. Thorax 1997;52:483-5.

4 Barnes PJ. NO or no NO in asthma? Thorax 1996;51:218-20.

5 Spina D. Airway sensory nerves: a burning issue in asthma? Thorax 1996;51:335-7.

6 Rogers DF. Scorpion venoms: taking the sting out of lung disease. Thorax 1996;51:546-8.

7 Dent G, Giembycz MA. Phosphodiesterase inhibitors: Lily the pink's medicinal compound for asthma? Thorax the pink's medic

8 Smith SF. Lipocortin 1: glucocorticoids caught in the act. Thorax 1996;51:1057-9.

9 Symon FA, Wardlaw AJ. Selectins and their counter receptors: a bitter sweet attraction. Thorax 1996;51:1155-7.

10 Page CP. Proteoglycans: the 'Teflon' of the airways? Thorax 1997;52:924-5.

11 Erjefält JS, Persson CGA. Airway epithelial repair: breathtakingly quick and multipotentially pathogenic. Thorax 1997;52:1010-2.

12 Nicholas TE, Doyle IR, Bersten AD. Surfactant replacement therapy in ARDS: white knight or noise in the system. Thorax 1997;52:195-7.

13 Pabst R, Tschernig T. Lymphocyte dynamics: caution in interpreting BAL numbers. Thorax 1997;52:1078-80.

14 Bingle L, Tetley TD. Secretory leukoprotease inhibitor: partnering $\alpha_{1}$-proteinase inhibitor to combat pulmonary partnering $\alpha_{1}$-proteinase inhibitor to

15 Tomee JFC, Koëter GH, Kauffman HF. Secretory leukoprotease inhibitor: a native antimicrobial protein presenting a new therapeutic option? Thorax 1998;53:114-6.

16 Adnot S, Raffestin B. Pulmonary hypertension: NO therapy? Thorax 1996;51:762-4.

17 Scott PH, Peacock AJ. Cell signalling in pulmonary vascular cells: do not shoot the messenger! Thorax 1996;51:864-6.

18 Lympany PA, duBois RM. Diffuse lung disease: product of genetic susceptibility and environmental encounters. Tho$\operatorname{rax} 1997 ; 52: 92-4$

19 Coker RK, Laurent GJ. Anticytokine approaches in pulmonary fibrosis: bringing factors into focus. Thorax 1997;52: 294-6.

20 Spurzem JR. Function at the junction: dynamic interactions between lung cells and extracellular matrix. Thorax 1996;51:956-8

21 Persson CGA. In vivo veritas: the continuing importance of discoveries in complex biosystems. Thorax 1996;51:441-3. 